\title{
Localized attentional interference reflects competition for reentrant processing
}

\author{
Kelly S. Steelman-Allen and Jason S. MCCARLey \\ University of Illinois at Urbana-Champaign, Urbana, Illinois \\ AND \\ JEFFREY R. W. MounTS \\ State University of New York, Geneseo, New York
}

\begin{abstract}
Visual performance is compromised when attention is divided between objects that are near one another in the visual field. It has been postulated that this effect, termed localized attentional interference (LAI), reflects competition between visual-object representations for the control of cortical neural responses. To determine whether LAI arises during feedforward processing or during reentrant processing, the present study examined the influence of poststimulus pattern and four-dot masks on the strength of the effect. Experiment 1 found that pattern masks, which are believed to compromise feedforward processing, do not produce stronger LAI than do four-dot masks, which are believed to leave feedforward processing undisrupted. Experiment 2 found that LAI is weaker when reentrant processing is interrupted shortly after initiation than it is when reentrant processing is allowed to run to completion. The results suggest that LAI emerges from competition between objects during reentrant processing.
\end{abstract}

The ability to divide attention between multiple objects is compromised when the attended items are near one another in the visual field. Judgments of the shapes of two objects, for example, become slower and more prone to error as the attended stimuli are moved closer together (see, e.g., Bahcall \& Kowler, 1999; Cutzu \& Tsotsos, 2003; McCarley, Mounts, \& Kramer, 2007). Similarly, perceptual judgments are compromised when a single to-be-attended item falls in the vicinity of an attentioncapturing distractor (see, e.g., Mounts, 2000). Such interference hinges on the observers' attentional set (Mounts, McCarley, \& Terech, 2007) and persists even when potential sensory confounds between stimuli are eliminated (Bahcall \& Kowler, 1999; Mounts \& Gavett, 2004). The effect thus appears to result from attention-driven processing and has been termed localized attentional interference (LAI).

The phenomenon of LAI accords well with the view of attentional selection as a competition between extrastriate object representations (Desimone \& Duncan, 1995; Luck, Girelli, McDermott, \& Ford, 1997). As visual signals progress from V1 through the extrastriate cortex, receptive fields (RFs) increase in size, reaching diameters large enough to encompass several objects simultaneously. As a result, the properties of multiple stimuli can be conflated in a neural response, preventing an accurate representation of any single item (Luck \& Beach, 1998). Modern accounts of attention hold that, to overcome this difficulty, object representations compete through mutual suppression for the control of neural responses (e.g., Desimone \& Duncan, 1995; Luck et al., 1997). An object becomes fully selected, then, when it dominates competing stimuli to assume control of a neural ensemble; effectively, the neurons' RFs constrict around the attended object, eliminating interference from surrounding items (Moran \& Desimone, 1985). It has been hypothesized that LAI reflects the competition that results as multiple to-be-attended items are moved closer together and therefore become more likely to fall within the same RFs (McCarley et al., 2007).

The precise nature and locus of LAI within the visualprocessing stream, though, have not been determined. Increases in RF size from early to later levels of the visual cortex are produced by a convergence of inputs from one region to the next within a pyramidal neural architecture (Tsotsos, 1990). At the same time, the visual cortex is rich with reentrant connections from higher to lower levels (Felleman \& Van Essen, 1991). Accordingly, psychophysical and electrophysiological data suggest that a preliminary feedforward sweep of activation allows rapid, but rudimentary, object and scene recognition (Thorpe, Fize, \& Marlot, 1996; VanRullen, 2007), but that reentrance is necessary for detailed perception (Lamme \& Roelfsema, 2000; Milner, 1974; Tsotsos, 1990). It has been postulated, therefore, that reentrance subserves a form of perceptual hypothesis testing, allowing the visual system to confirm or resolve a coarse preliminary representation 
by reconciling it with the ongoing stream of feedforward signals (Di Lollo, Enns, \& Rensink, 2000). Feedforward and reentrant mechanisms also differ in that feedforward operations occur in parallel and without capacity limitations (Rousselet, Fabre-Thorpe, \& Thorpe, 2002), whereas reentrant analysis demands limited-capacity attentional processing (Di Lollo et al., 2000). Experiments by Enns (2004) demonstrated that feedforward and reentrant processing can be distinguished through poststimulus masking, with the disruption of feedforward processes manifesting as visual degradation that occurs at short stimulus onset asynchronies (SOAs) between target and mask, and the disruption of reentrant processes appearing as degradation that occurs even at long SOAs, but only when attention is not focused on the target.

Neurophysiological data, notably, tie the competitiveinteraction account of attention to the feedforwardreentrant distinction. When two objects, one preferred and one nonpreferred, are presented simultaneously within a neuron's RF, the cell's initial response is a wave of activation that is the same regardless of which object is attended. Only after a 100- to 200-msec delay does attentional modulation emerge, with the cell's response level increasing if the preferred stimulus is attended, and decreasing if the nonpreferred item is attended (Chelazzi, Miller, Duncan, \& Desimone, 1993). These results suggest that top-down control of competition is effected by feedback from higher regions to the extrastriate cortex (Desimone \& Duncan, 1995).

The goal of the present study was to examine the place of LAI within the cycle of feedforward and reentrant processing. A feedforward model of the effect might attribute LAI to the perceptual degradation that occurs when the properties of multiple objects are conflated in the first sweep of activation following stimulus onset. By such an account, attentional modulations that occur in single-cell responses following the feedforward sweep would resolve the interference reflected by LAI. Such a model seems inconsistent, however, with the finding that LAI is strongly modulated by the observer's set for features such as color and shape (e.g., Mounts et al., 2007); as noted, single-cell data indicate that the initial response following stimulus onset is independent of attentional set for particular object properties (Chelazzi et al., 1993; Motter, 1994). Within a reentrant account, alternatively, LAI presumably reflects the interactions by which an object's properties are disambiguated following the feedforward sweep. When a pair of to-be-attended objects are near one another, reentrant processes responsible for disambiguating them would compete for control of shared RFs, producing reciprocal interference and poor perceptual performance. As the stimuli are moved farther apart, reentrant competition for RFs would be reduced, and perceptual performance would improve. LAI would thus be a psychophysical consequence of variations in the quality of reentrant processing.

To distinguish between these models, the present experiments examined the effects of poststimulus pattern and four-dot masks on the magnitude of LAI. Masks are illustrated in Figure 1. Psychophysical (Di Lollo et al., 2000; Enns, 2004; Enns \& Di Lollo, 1997), neurophysiological (Woodman \& Luck, 2003), and computational (Francis \&
A

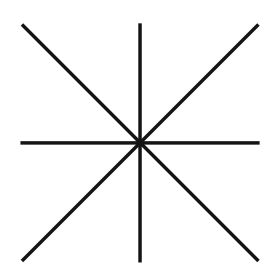

Figure 1. Examples of the pattern (A) and four-dot (B) masks used in Experiment 1.

Cho, 2007) evidence indicates that pattern and four-dot masks have qualitatively different effects on perceptual processing. Both forms of mask appear to disrupt perception through a process of object substitution that occurs when a target stimulus is replaced by a mask before reentrant processing has resolved the target representation. In such cases, reentrant processes triggered by the target encounter incoming feedforward signals inconsistent with the preliminary target representation, and the target is therefore displaced from the perceptual stream without entering consciousness (Enns \& Di Lollo, 1997; Woodman \& Luck, 2003). In contrast, pattern, but not four-dot, masks also appear to disrupt feedforward processing through temporal integration of target and mask contours; four-dot masks, because they contain very little contour and are generally designed not to overlap the target object, appear to leave feedforward processing undisturbed, selectively disrupting reentrant processes (Enns, 2004). Given these assumptions, feedforward and reentrant accounts of LAI predict different effects of the two forms of mask, dependent on target-mask SOA. If LAI arises during the feedforward sweep and is resolved by reentrant processing, it should be stronger at short SOAs, before reentrant processes have been allowed to resolve the interference between objects. Additionally, because interference at small separations should increase the time needed for feedforward processes to achieve a preliminary object representation, masks that degrade feedfoward processing should produce stronger LAI than do masks that selectively compromise reentrant processes. At short target-mask SOAs, therefore, pattern masks should allow stronger LAI than do four-dot masks. At long SOAs, after feedforward processes have run their course, pattern and four-dot masks should have equivalent effects. Conversely, if LAI arises during reentrance, then the effect should be weak immediately following stimulus onset and grow stronger at long SOAs, after reentrant processing has begun. Furthermore, the strength of the effect should be the same with four-dot masks and pattern masks at all SOAs, since both forms of mask will interrupt reentrant processing. ${ }^{1}$

\section{EXPERIMENT 1}

Experiment 1 employed a partial-report task (Townsend \& Ashby, 1983) modeled after experiments by Enns (2004) to determine whether LAI has its locus in feedforward or reentrant processing. On each trial, participants saw a ring 


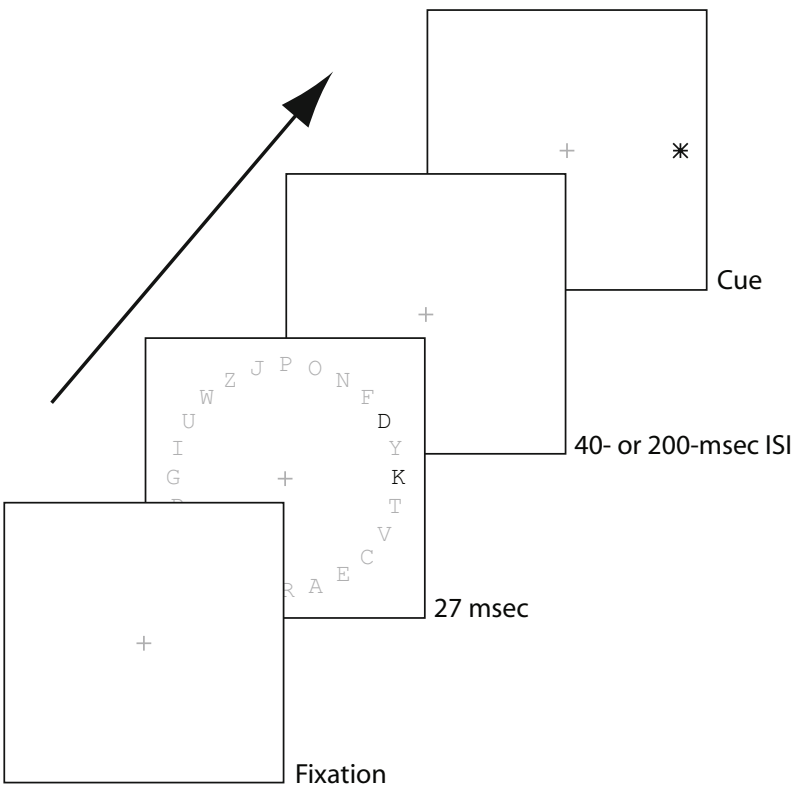

Figure 2. Stimuli and procedure of Experiment 1. Black letters represent attended red stimuli, and gray letters represent unattended gray fillers. All characters were matched in luminance contrast within actual stimulus displays.

of letters, two of them red and the rest gray. The stimuli remained visible briefly and were followed by either a pattern or a four-dot mask after an interstimulus interval (ISI) of either 40 or $200 \mathrm{msec}$. The mask appeared at the location of one of the two red items, and the participants' task was to report the identity of the masked item. The poststimulus mask thus served as a cue that indicated which of the items to report. Since there was no way to know which of the two red letters to report until the mask appeared, participants were required to attend to both red items while they were visible. The effects of LAI were gauged by varying the spatial separation between the red letters.

\section{Method}

Participants. The participants were 22 young adults ( 7 female, 15 male, mean age $=20.5$ years) with normal or corrected acuity and with normal color vision.

Apparatus and Stimuli. The stimuli were presented on a 22 -in. monitor with a refresh rate of $75 \mathrm{~Hz}$, and they were viewed from a distance of $77 \mathrm{~cm}$, controlled by a cheekrest. The stimuli were 24 upright capital letters; were randomly selected without replacement from the English alphabet, excluding Q; and were presented in either gray or red on a black background. Letters were drawn in 40-pt Courier font with dimensions of $0.67^{\circ} \times 0.60^{\circ}$, and they were spaced evenly about an imaginary circle of radius $5.87^{\circ}$ that was centered on a gray fixation cross of $0.74^{\circ} \times 0.74^{\circ}$. On each trial, two letters were drawn in red, and the rest were drawn in gray. Two types of masks were used: a four-dot mask and a pattern mask. Four-dot masks comprised four circles $0.06^{\circ}$ in radius, each one centered and contained on one of the corners of an imaginary square that measured $0.81^{\circ} \times$ $0.81^{\circ}$. Pattern masks were eight-pointed asterisks that were centered within the same imaginary square. Masks were presented in red. All stimuli were approximately $10 \mathrm{~cd} / \mathrm{m}^{2}$ in luminance.

Procedure. The procedure is illustrated in Figure 2. Each trial began with the display of a fixation cross. The fixation display remained visible until the participant made a keypress. After a 13-msec delay, stimulus letters appeared for a duration of $27 \mathrm{msec}$, followed by either a 40- or a 200-msec ISI, after which the target letter was masked. The SOA between target and mask was therefore either 67 or $227 \mathrm{msec}$; these values, chosen with the goal of interrupting feedforward processing in one case (short SOA) and allowing it to reach completion in the other (long SOA), were selected on the basis of Enns's (2004) findings and were pilot tested with the present task. The participants' task was to report the identity of the masked letter. The mask remained visible until a response was made. Participants were instructed to respond as accurately as possible and, if they were uncertain, to guess. Responses were unspeeded.

Separation between red items varied from one to six positions. For a given trial, the positions of the two red items were selected randomly, under the constraint that the separation between them matched the value specified for that trial. Observers performed one practice block of 24 trials and five experimental blocks of 48 trials with each form of mask. Each block contained an equal number of trials for all values of separation between red items. The order of the warm-up blocks was random, as was the order of the experimental blocks. The order of trials was randomized within blocks.

\section{Results}

Mean error rates are presented in Figure 3. Data were analyzed with a $2 \times 2 \times 6$ ANOVA, using mask (pattern vs. four dot), SOA (67 vs. $227 \mathrm{msec}$ ), and separation between attended items (1-6 positions) as within-subjects factors. Results confirmed that pattern masks were more effective in general than four-dot masks $\left[F(1,21)=63.289, M S_{\mathrm{e}}=\right.$ $.013, p<.001]$ and that error rates were lower at the long SOA than at the short SOA $\left[F(1,21)=737.929, M S_{\mathrm{e}}=\right.$ $.025, p<.001]$. As anticipated, however, these effects were qualified by a mask $\times$ SOA interaction $[F(1,21)=$ $\left.49.244, M S_{\mathrm{e}}=.010, p<.001\right]$, indicating that differences between the strength of pattern masks and that of fourdot masks obtained only at the short SOAs. The results were therefore consistent with the expectation that pattern masks, by compromising feedforward processing, would be more effective than four-dot masks at short SOAs but not at long SOAs. Data also showed a reliable main effect of separation $\left[F(4.32,90.72)=10.926, M S_{\mathrm{e}}=.010\right.$,

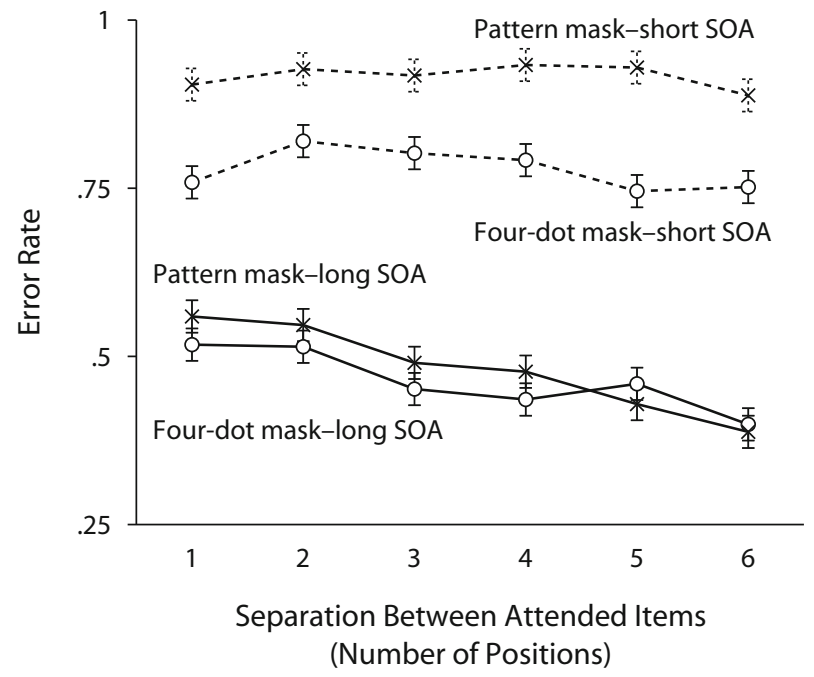

Figure 3. Error rates of Experiment 1. Error bars represent \pm 1 within-subjects standard error (Loftus \& Masson, 1994) on the basis of the mask $\times$ ISI $\times$ distance interaction. 
$p<.001]$ qualified by an SOA $\times$ separation interaction $\left[F(3.96,83.15)=6.482, M S_{\mathrm{e}}=.010, p<.001\right]$, indicating that error rates declined as the distance between attended items increased and that the effect of distance was larger at the longer SOA. ${ }^{2}$ As predicted by a reentrant account of LAI, therefore, competition between attended items increased with time following stimulus onset. Finally, data showed no reliable mask $\times$ separation $[F(3.90,81.89)=$ $\left.0.305, M S_{\mathrm{e}}=.010, p=.870\right]$ or mask $\times \mathrm{SOA} \times$ separation $\left[F(4.07,85.56)=1.503, M S_{\mathrm{e}}=.013, p=.208\right]$ interaction, giving no evidence that pattern masking increased the strength of LAI.

\section{Discussion}

The results of Experiment 1 provide two pieces of evidence that LAI occurs during reentrant, not feedforward, processing. First, spatial interference between attended items was greater at the long SOA, indicating that LAI was weak or absent in the earliest wave of processing. Although this result does not wholly preclude a feedforward account of LAI - it might be reconciled, for example, with a model in which interference occurs late in the feedforward sweep - it is predicted by a reentrant-processing account. Second, the strength of LAI was not exacerbated by pattern masks, which are believed to compromise feedforward processing at short SOAs (Enns, 2004). Under a feedforward model of LAI, masks that terminated feedforward processing early should have produced a stronger gradient of interference than did masks that allowed the feedforward sweep to run to completion. The null mask $\times$ distance and mask $\times$ SOA $\times$ distance interactions therefore imply that spatial interference between attended items arose in reentrant processing. Experiment 2 tested this conclusion further.

\section{EXPERIMENT 2}

The results of Experiment 1 suggest that LAI is no stronger when feedforward processing is disrupted than when it is not, implying that spatial interference between attended items emerges in reentrant processing. Experiment 2 was designed to seek converging evidence for this conclusion. If LAI is produced by reentrant competition between object representations, then the effect should be stronger when reentrant processes run to completion than when they are terminated very soon after their initiation. To test this prediction, the present experiment compared the strength of LAI under conditions of short-SOA four-dot masking and no masking. Because there was no mask to use as a partialreport cue in the unmasked conditions, the experimental task was modified slightly from that of the first experiment. Here, the stimuli were similar to those of Experiment 1, except that one of the two red characters on each trial was a digit instead of a letter, and the participants' task was to report the identity of the red letter.

\section{Method}

Participants. The participants were 22 young adults ( 11 female, 11 male, mean age $=18.5$ years) with normal or corrected acuity and with normal color vision.

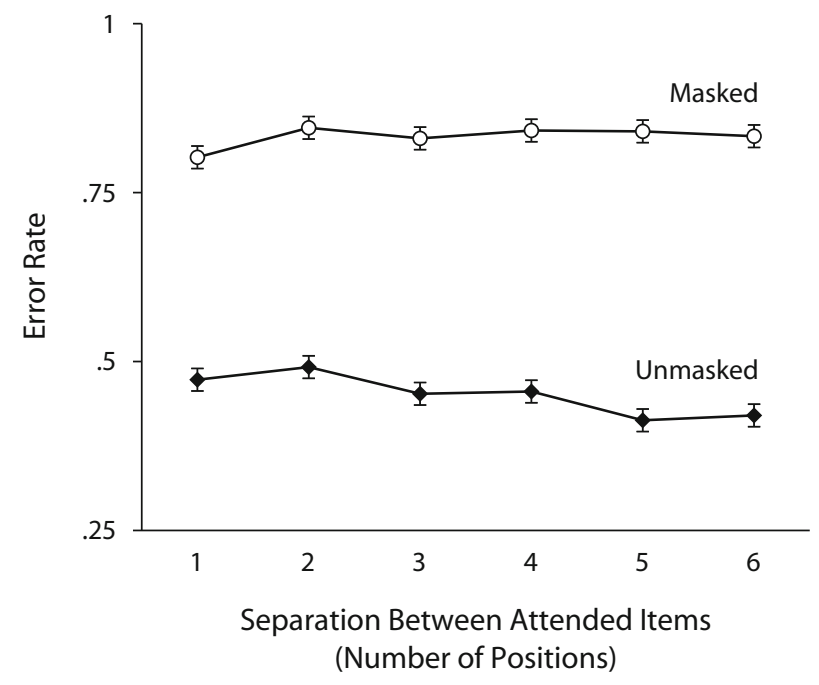

Figure 4. Error rates of Experiment 2. Error bars represent \pm 1 within-subjects standard error on the basis of the mask $\times$ distance interaction.

Apparatus and Stimuli. The apparatus and stimuli were identical to those of Experiment 1, except that one of the two red items presented in each trial was a randomly selected digit between 1 and 9 .

Procedure. The procedure was identical to that of Experiment 1, with the following exceptions. First, the pattern-mask conditions of the first experiment were replaced with no-mask conditions. Second, the participants' task was to identify the single red letter in each trial. Third, in the masked condition, a single SOA of $67 \mathrm{msec}$ was used, chosen on the basis of the data of Experiment 1 and with the aim of preempting reentrant processes shortly after their onset.

\section{Results}

Figure 4 presents mean error rates for target identification. For analysis, data were submitted to a $2 \times 6$ ANOVA, using mask (four dot vs. no mask) and separation between attended items (one to six positions) as within-subjects factors. As expected, the results confirmed that error rates were higher under masked conditions than under unmasked conditions $\left[F(1,21)=317.477, M S_{\mathrm{e}}=.030\right.$, $p<.001]$ and that error rates declined as separation between attended objects increased $[F(4.19,87.89)=2.586$, $\left.M S_{\mathrm{e}}=.051, p=.040\right]$. Finally, and importantly, a reliable interaction $\left[F(3.73,78.38)=3.206, M S_{\mathrm{e}}=.006, p=\right.$ $.019]$ indicated that LAI was stronger under unmasked conditions than under masked conditions. One-way ANOVAs that were conducted separately for the masked and unmasked conditions showed a reliable effect of separation in the latter $\left[F(4.29,90.13)=3.44, M S_{\mathrm{e}}=.007, p=\right.$ $.01]$ but not in the former $\left[F(3.96,83.12)=1.86, M S_{\mathrm{e}}=\right.$ $.004, p=.126]$. The results suggest that LAI is greater when reentrant processes run to completion than when they are terminated shortly after onset. ${ }^{3}$

\section{GENERAL DISCUSSION}

A pair of experiments examined the influence of pattern and four-dot masks on the strength of localized interference between a pair of attended items. Experiment 1 
found that LAI was stronger at a 227 -msec target-mask SOA than at a $67-\mathrm{msec}$ SOA but was equivalent for pattern and four-dot masks in both cases. Experiment 2 found that LAI was stronger when reentrant processes were allowed to run to completion than when they were terminated shortly after initiation. Together, these findings imply that LAI does not reflect interference in the feedforward sweep but instead results from competition that occurs between object representations during reentrance. Couched more specifically within the theory of reentrance offered by Di Lollo et al. (2000), the data suggest that proximity between attended items produces noise or ambiguity in the process of reentrant perceptual hypothesis testing. As noted earlier, Di Lollo and colleagues postulated that reentrant processes seek correspondences between high- and low-level signals as a way to disambiguate high-level object representations that are achieved by the feedforward sweep. Within this account, LAI might result when reentrant projections from a highlevel representation encounter low-level signals representing multiple objects. Phrased differently, LAI may result when multiple attended items fall within the same reentrant RFs, compromising the reentrant processes' ability to resolve any single object representation.

Notably, the conclusion that LAI occurs during reentrance is consistent with earlier findings about both LAI and four-dot masking. Woodman and Luck (2003), for example, reported that the neural processing of rudimentary shape information survives four-dot masking and thus does not appear to require reentrant signaling. Correspondingly, McCarley and Mounts (2007) found no LAI in a feature-detection (shape or color) task. Rather, the effect emerged only when observers were asked to resolve individual objects. In line with the present data, these findings imply that the feedforward sweep allows detection of coarse feature information, and that LAI may obtain only when a demand to resolve and conjoin the properties of individual objects (cf. Treisman \& Gelade, 1980) necessitates reentrance. Experiments by Jiang and Chun (2001) have also demonstrated that the strength of four-dot masking declines gradually with distance from the mask. This suggests that the reentrant resources revealed by four-dot masking are spatially extended, which means that competition for these resources could well produce the spatially graded interference that was seen in LAI.

\section{AUTHOR NOTE}

Correspondence concerning this article should be addressed to J. S. McCarley, Beckman Institute, 405 N. Mathews Avenue, Urbana, IL 61801 (e-mail: mccarley@illinois.edu).

\section{REFERENCES}

BAHCALL, D. O., \& Kowler, E. (1999). Attentional interference at small spatial separations. Vision Research, 39, 71-86.

Chelazzi, L., Miller, E. K., Duncan, J., \& Desimone, R. (1993). A neural basis for visual search in inferior temporal cortex. Nature, 363, 345-347.

Cutzu, F., \& Tsotsos, J. K. (2003). The selective tuning model of attention: Psychophysical evidence for a suppressive annulus around an attended item. Vision Research, 43, 205-219.
Desimone, R., \& Duncan, J. (1995). Neural mechanisms of selective visual attention. Annual Review of Neuroscience, 18, 193-222.

Di Lollo, V., EnNs, J. T., \& RensinK, R. A. (2000). Competition for consciousness among visual events: The psychophysics of reentrant visual process. Journal of Experimental Psychology: General, 129, 481-507.

ENNS, J. T. (2004). Object substitution and its relation to other forms of visual masking. Vision Research, 44, 1321-1331.

EnNs, J. T., \& Di Lollo, V. (1997). Object substitution: A new form of masking in unattended visual locations. Psychological Science, 8, 135-139.

Felleman, D. J., \& Van Essen, D. C. (1991). Distributed hierarchical processing in the primate cerebral cortex. Cerebral Cortex, 1, 1-47.

Francis, G., \& CHO, Y. S. (2007). Testing models of object substitution with backward masking. Perception \& Psychophysics, 69, 263-275.

JIANG, Y., \& CHUN, M. M. (2001). The spatial gradient of visual masking by object substitution. Vision Research, 41, 3121-3131.

Kristjansson, A., \& Nakayama, K. (2002). The attentional blink in space and time. Vision Research, 42, 2039-2050.

LAmme, V. A., \& Roelfsema, P. R. (2000). The distinct modes of vision offered by feedforward and recurrent processing. Trends in Neurosciences, 23, 571-579.

Loftus, G. R., \& Masson, M. E. J. (1994). Using confidence intervals in within-subjects designs. Psychonomic Bulletin \& Review, 1, 476-490.

LuCK, S. J., \& BEACH, N. J. (1998). Visual attention and the binding problem: A neurophysiological perspective. In R. D. Wright (Ed.), Visual attention (pp. 455-478). Oxford: Oxford University Press.

Luck, S. J., Girelli, M., McDermott, M. T., \& Ford, M. A. (1997). Bridging the gap between monkey neurophysiology and human perception: An ambiguity resolution theory of visual selective attention. Cognitive Psychology, 33, 64-87.

McCarley, J. S., \& Mounts, J. R. W. (2007). Localized attentional interference affects object individuation, not feature detection. Perception, 36, 17-32.

McCarley, J. S., Mounts, J. R. W., \& Kramer, A. F. (2007). Spatially mediated capacity limits in attentive visual perception. Acta Psychologica, 126, 98-119.

Milner, P. M. (1974). A model for visual shape perception. Psychological Review, 81, 521-535.

Moran, J., \& Desimone, R. (1985). Selective attention gates visual processing in the extrastriate cortex. Science, 229, 782-784.

MotTER, B. C. (1994). Neural correlates of attentive selection for color or luminance in extrastriate area V4. Journal of Neuroscience, $\mathbf{1 4}$ 2178-2189.

Mounts, J. R. W. (2000) Evidence for suppressive mechanisms in attentional selection: Feature singletons produce inhibitory surrounds. Perception \& Psychophysics, 62, 969-983.

Mounts, J. R. W., \& GaVett, B. E. (2004). The role of salience in localized attentional interference. Vision Research, 44, 1575-1588.

Mounts, J. R. W., McCarley, J. S., \& Terech, A. M. (2007). Attentional templates regulate competitive interactions among attended visual objects. Perception \& Psychophysics, 69, 209-217.

Rousselet, G. A., Fabre-Thorpe, M., \& Thorpe, S. J. (2002). Parallel processing in high-level categorization of natural images. Nature Neuroscience, 5, 629-630.

Thorpe, S. J., Fize, D., \& Marlot, C. (1996). Speed of processing in the human visual system. Nature, 381, 520-522.

Townsend, J. T., \& Ashby, F. G. (1983). Stochastic modeling of elementary psychological processes. Cambridge: Cambridge University Press.

TownsEnd, J. T., \& LANDON, D. E. (1982). An experimental and theoretical investigation of the constant-ratio rule and other models of visual letter confusion. Journal of Mathematical Psychology, 25, 119-162.

Treisman, A. M., \& Gelade, G. (1980). A feature-integration theory of attention. Cognitive Psychology, 12, 97-136.

Tsotsos, J. K. (1990). Analyzing vision at the complexity level. Behavioral \& Brain Sciences, 13, 423-469.

VANRULlen, R. (2007). The power of the feed-forward sweep. Advances in Cognitive Psychology, 3, 167-176.

Woodman, G. F., \& LuCK, S. J. (2003). Dissociations among attention, perception, and awareness during object-substitution masking. Psychological Science, 14, 605-611. 


\section{NOTES}

1. At least two studies (Kristjansson \& Nakayama, 2002; Mounts, 2000) have examined the time course of spatial-attentional interference, but neither included a manipulation to distinguish feedforward from reentrant mechanisms.

2. One concern in interpreting the $\mathrm{SOA} \times$ distance interaction is that the mean error rate was lower at the long SOA, which means that differences in the strength of LAI might have resulted from quantitative differences in task difficulty rather than from qualitative differences between feedforward and reentrant processing. A control analysis tested this possibility. To begin, data were collapsed across experimental conditions and participants to form a confusion matrix of stimulus letters. A $2 \times 2$ submatrix was then extracted for every pair of letters, and the confusion rate for each pair was calculated (assuming that of the constant-ratio rule was applicable; Townsend \& Landon, 1982). Finally, a median split was performed on the letter pairs on the basis of confusability, and data were submitted to a within-subjects ANOVA with confusability and separation between attended items as factors. The mean error rate was .568 for high-confusability stimuli and .389 for low-confusability stimuli. Analysis revealed no reliable interaction $\left[F(3.56,75.51)=1.673, M S_{\mathrm{e}}=\right.$ $.003, p=.148]$, however, suggesting that the magnitude of LAI did not differ as a function of general task difficulty.

3. A control analysis like that of Experiment 1 again showed no reliable interaction of confusability and separation (the mean error rate was .561 and .336 for high- and low-confusability letter pairs, respectively) $\left[F(3.99,83.73)=1.11, M S_{\mathrm{e}}=.003, p=.358\right]$.

(Manuscript received June 2, 2008; revision accepted for publication July 28,2008 .) 